\title{
Using a Hematology Curriculum in a Web Portfolio
} Environment

\section{Fredrik Enoksson*}

Royal Institute of Technology and Uppsala University

Lindstedtsvägen 3, floor 6

10044 Stockholm, Sweden

E-mail: fen@csc.kth.se

\section{Ambjörn Naeve}

Royal Institute of Technology and Uppsala University

Lindstedtsvägen 3, floor 6

10044 Stockholm, Sweden

E-mail: amb@csc.kth.se

\section{Eva Hellström-Lindberg}

Karolinska Institutet

Department of Medicine, Division of Hematology

Karolinska University, Hospital Huddinge

14186 Stockholm, Sweden

E-mail: Eva.Hellstrom-Lindberg@ki.se.

*Corresponding author

\begin{abstract}
In 2005 the European Hematology Association developed the European Hematology Curriculum. This was distributed as a printed booklet and the intention was that junior hematologist could use it for personal competence development. In the EU-funded project H-net this Curriculum has been adapted into the a web environment by using RDF and placed inside a web portfolio system. How this is done is further described in this article. Furthermore, the possibilities of reusing the curriculum in ways that was not initially intended is described, such as describing Learning Resources inside the web-portfolio system with how they relate to different parts of the curriculum. That way a search for learning resources inside the portfolio by using the curriculum is enabled. And, since the medical field of hematology is closely related to other medical fields the design of the web-version of the curriculum was done in a way that builds for possible combination with any other curriculum in another medical field.
\end{abstract}

Keywords: Hematology; Competence; Learning resources; Curriculum; Portfolio system; Metadata

Biographical notes: Fredrik Enoksson is a PhD-student at the Knowledge Management Research group, KTH, Sweden and a research engineer at the Uppsala Learning Lab at the University of Uppsala. His research is focused on metadata description for Learning Resources in a web environment. 
Dr. Ambjörn Naeve is head of the Knowledge Management Research group at the Royal Institute of Technology in Stockholm and scientific director at Uppsala Learning Lab at Uppsala University. He is editor-in-chief of the International Journal of Technology Enhanced Learning, and co-chair of the World Summit on the Knowledge Society.

Eva Hellström-Lindberg holds a $\mathrm{MD}$ and a $\mathrm{PhD}$, and is professor of hematology at Karolinska Institutet and a specialist in hematology. Her research focuses on malignant hematological disorders. She is principal investigator of the pan-European project on training in hematology, H-Net.

\section{Introduction}

Hematology can be defined as "the branch of medicine that deals with diseases of the blood and blood-forming organs" (Hematology, 2006). However, several other definitions and description do exist ${ }^{1}$ and a hematologist is a physician who is specialised in hematology. According to the European Hematology Association (2008) the specialist training in Europe to become a hemtologist has shown to be heterogenous in aspects such as length and content of the training. The explanation for this difference can sometimes be found in the history of hematology in a region or a country (Bulgarian Society of Clinical and Transfusion Haematology, 2005) and the underlying reason behind can be found in that "Because of the nature of blood, the science of hematology profoundly affects the understanding of many diseases" (Hematology, 2003). Thus, the lines between hematology and other medical specialities have been drawn differently in different countries.

Most European countries have a national society for hematology or a national organisation where hematology is included. On the European level the European Hematology Association (EHA) works together with the hematologists of Europe and their national societies to achieve their mission: “.... to promote excellence in clinical practice, research and education in European hematology" as stated in (EHA, 2006). EHA are also working to make the training in hematology less heterogenous and a first step towards that was taken in 2005 when EHA and the national societies of hematology in Europe developed a European curriculum for hematology, that resulted in the EHA Curriculum Vitae Passport (in the rest of this article refererred to as the "CV-passport" or the "passport") for Hematology in Europe. This version of the passport was made available as a printed booklet, but in the EU-funded project $\mathrm{H}$-net ${ }^{2}$ a first version for the web has been developed. How that have been utilized is described further in this article.

\subsection{EHA CV-passport, a competence profile for hematology}

The purpose of developing the CV-passport was to make a common curriculum for European hematologists that they easily could make use of for personal competence development. It covers the essential parts of the field for a junior specialist in hematology and has been endorsed by all the national hematology societies in Europe (European

1 An example of this "The field of Hematology includes the study of blood forming elements, blood constituents, coagulation, and/or organs involved in, or that interact with, these physiologic functions" (Gitlin \& Melnick, n.d.)

2 http://www.hematologynet.eu 
Hematology Association, 2005). As all medical fields, hematology is evolving as procedures and routines are updated. For example, some tasks that previously were performed by the practising doctor are now only performed in certified laboratories. Therefore the CV-passport should be periodically evaluated and revised.

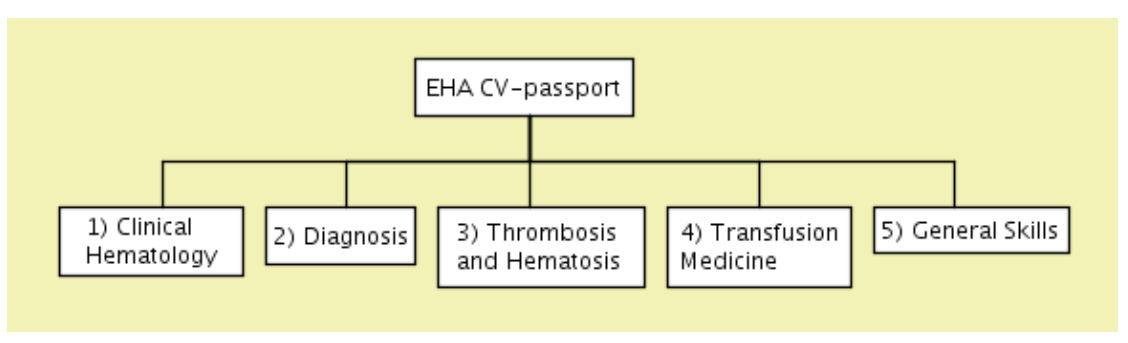

Figure 1. Overview of the CV-passport main sections

Currently the CV-passport consists of around 280 items, each of which describe a specific competence. These items are organised into a structure of 5 main sections, see figure 1, which in turn holds around 6-7 subsections each. Every item holds a description of what knowledge/competence it covers. This could for example be a specific disease or interpretation of some laboratory result. When a hematolgist fills out the passport an item gets a value that expresses the level of knowledge/competence of the hematologist. The possible values are limited to: "awareness", "knowledge" and "competence" and one item can only have one of these values. These values are defined as indicating.

- $\quad$ awareness: basic notions

- knowledge: updated concepts on patho-physiology, epidemiology, diagnosis, prognosis, and different therapeutic approaches.

- competence: adequate understanding and practical integration of knowledge and skills for optimal diagnosis and treatment of the patients at any phase of their disease.

These definitions are copied from the CV-passport (European Hematology Association, 2005) and meant for an audience with medical education. The values correspond to levels, where "awareness" is the lowest and "competence" the highest. This means that when the value is "competence" the levels of "knowledge" and "awareness" have already been reached. To simplify the definition of the values for people without medical knowledge one can say that the level "competence" for an item only can be considered to be achieved when the hematologist has had an actual case that dealt with what is contained in the description of an item. The possible values for an item will in the rest of this article be referred to as an "item value".

When put to use, the CV-passport can be seen as a competence profile of a hematologist. In the printed version of the passport the value on each item is filled out by hand. With the increasing use of the World Wide Web with all the different kinds of applications, a version of the passport that is available on the Web would be beneficial in several aspects, where some of them are listed below:

1. Ease of use and accessibility: The passport would be possible to access and update using a normal web browser. It would also be possible to store the passport locally on a computer or mobile device.

2. Combining with other medical fields: With a digital CV-passport made in a similar way for another field of speciality, a combination of the two would be 
possible.

3. Statistics: Gathering of data would become easier as the data is already in a digital format that can be processed by a computer.

4. Development of learning goals: this goal is set by a junior hematologist by selecting a set of items and for each one of them set a desired level. The differences between the levels for each item and the actual current level is called a competence gap. This can be facilitated by an application using the digital CVpassport.

5. Personalised searches of learning resources: A kind of search that takes the competence gap into account as proposed by Naeve, Sicilia, and Lytras (2008). This would require that information on how a learning resource relates to learning goals, which is made possible with the CV-passport.

6. Possible to tag learning resources: With resources tagged by using the sections, subsections or items of the CV-passport searches can be done on learning resources that deals with a certain section or item of the passport. An similar approached is suggested by Van Assche (2007).

7. Social web: The CV-passport could be reused in Social web applications where learning goals could be explored and developed.

Some of these aspects have been considered further and developed in H-net project, and in the following sections the first web-version of the CV-passport is described and discussed along with the surrounding technical environment and what possibilities it opens up for.

\section{Web-portfolios for Hematology}

In order for the digital version of the $\mathrm{CV}$-passport to be utilized, an integration into a surrounding environment is needed. In $\mathrm{H}$-net this environment is a system of personal web-portfolios built upon a generic framework for portfolios called SCAM and which is described in more detail by Hebner and Palmér (2009). This system offers many of the features a portfolio-system usually includes, but with the integration of the CV-passport each user has a possibility to store it as part of the personal profile.

The integration has also considered how learning resources uploaded to or linked from the portfolio can be semantically "tagged" to how they relate to one or more items in the CV-passport. By making use of the passport a personalised search for educational content based on a competence gap is also made possible. These are some of the aspects that makes the portfolio system extra useful for the hematology community and that will be further described in the following chapters. In order to make this possible together with the aspects listed at the end of the previous chapter, the choice of technology becomes important. 


\subsection{Technical aspects}

The portfolio system is a web-application that can be accessed using a modern webbrowser. It makes heavy use of Javascript for layout and AJAX ${ }^{1}$-technology for communication between the browser and the server. The principles of REST (Fielding, 2000) are also followed as much as possible in order for the system to comply with how the WWW works.

These technical choices have been based upon how the WWW seems to evolve with more and more possibilities to reuse data-sources in different and unexpected situations. A typical situation where this is done are so called mash-ups or widgets that can combine different data sources and then choose how to present the resulting data with different kind of styling techniques. The portfolio system works in a similar way where all layout and style information is done in the client/browser and the information communicated between the client and the server is basically just data. This also leaves treatment of the data up to the client, and the web-portfolio system becomes just one out many possible applications that can use this data.

Another aspect of reusing data in different contexts is the question about interoperability. In order to achieve interoperability, a standard for representing data is needed, and preferably a standard that is used by many stakeholders. XML is often used when exchanging data, but that is more of a syntax for the data. Instead the system makes use of the metadata framework RDF (Miller and Manola, 2004), that has been developed by the World Wide Web Consortium ${ }^{2}$. It has a flexible data model that makes it easy to extend the data, so what can be represented in the data does not need to be decided beforehand. Furthermore, the data is not dependent on a specific syntax, so the same data can be expressed in many ways, for example in XML. When requests are sent to the portfolio-server the wanted syntax can be specified. By default the data is returned in XML syntax. For communication between the portfolio web-client and the server a format called $\mathrm{JDIL}^{3}$ is used which is a version of $\mathrm{JSON}^{4}$.

\subsection{Digital version of the $\mathrm{CV}$-passport}

As previously stated, the CV-passport is structured in a hierarchy with section and subsections that holds the items. No relations or dependencies across the hierarchy were found that was necessary to include when modelling the data-representation. It was important to try to strive for simplicity so that other similar passports in the future could be combined with this one ${ }^{5}$. An example of that would be if the closely related medical field of oncology were to create a similar CV-passport, it should be possible to reuse items from the hematology $\mathrm{CV}$-passport.

Since the digital version of the CV-passport should be possible to reuse in different web environments, it is expressed as an RDF model and is making use of some established RDF-vocabularies. How parts of the passport are modelled can be seen in Figure 2 below, which shows how an item is represented and how it relates to a subsection and further on to a main-section. The concepts and concept-relations (called

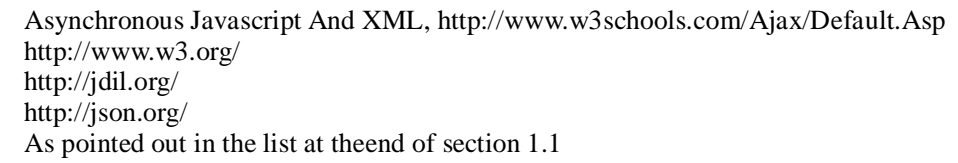


properties in RDF) are identified with a URI, which is a unique identifier for the Internet. In the figure they are written in an abbreviated form with a so called namespace to the left of the colon-symbol. It can from the namespace be concluded which RDF-vocabulary has been used in the model. The namespace skos is a vocubulary used for simple knowledge organisation (Summers \& Isaac, 2009) and is here used to form the hierarchy present in the passport. The ellipse in the top left corner represents the whole CVpassport and has the namespace $e h a$ that is especially made for the passport. This ellipse points out the main sections by using the relation skos:member, which is used then used to further build the hierarchy with subsections and at the end the items. In figure 2 a part of this is shown with the main section Clinical Hematology, subsection Red Cell Disorders and the item called 1AA.

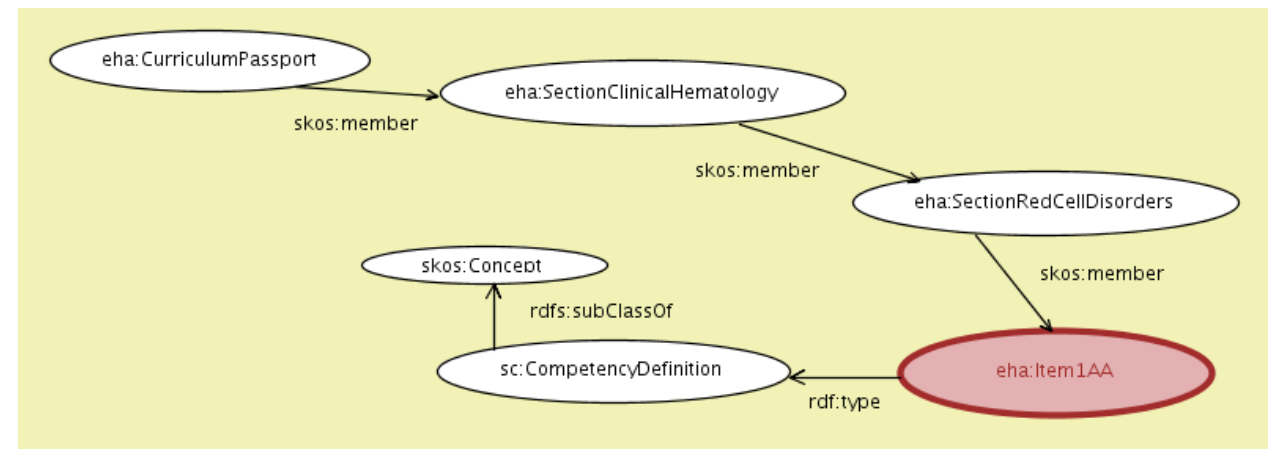

Figure 2. Example of the CV-passport structure and an item

The only outgoing relation an item has here is its classification by the relation rdf:type, which is taken from the RDF Schema that is a vocabulary to be used for creating RDF-vocabularies and it uses both the namespaces $r d f$ and $r d f s$. All items are classified as being a Competency-definition, where the namespace $s c$ is one used for the portfolio system. This was because no real well established and well-maintained RDF-vocabulary exists yet for competence-description. An approach that fits with the CV-passport is found in Schmidt and Kunzmann (2006) which is described more in chapter 3.2. An important feature here is that the item does not point out what subsection it belongs to, instead the sub-section points to its members. If we again consider aspect $\mathrm{nr} 2$ in the list at the end of chapter 3.1, ie building for easy combination with other passport, it becomes easy to point out a specific item by using its URI. Hence, other passport can point to a specific item by using its URI, and the information on what sections that uses the item is maintained at each passport.

In the portfolio system a user can fill out the his/her own passport and store that as part of the profile information. A screenshot of this editor is shown in figure 3 which also shows a part of the passport. Here the structure of the passport is only used to organize the items in the sections for the editor and the information stored about the user disregards this. How this information is structured is shown in figure 4 where the URI identifying the user can be found at the top. Below this URI an instance of a competenceprofile with its own URI can be found. As can be seen the competence-profile points out the user of which competence it describes with the relation sc:aboutPerson. The relation is in that direction is due to a technical solution in the portfolio-system that makes it easy to hide the competence-profile from other users, otherwise it would have seen more natural to have the competence-profile being pointed out from the user. The competenceprofile uses the relation sc:hasCompetence for each item that has been filled it by the 
editor. The relation needs to point to a combination of an item and an item-value, and as this combination does not need to be identified outside of the scope of the description it is not assigned a URI. This is in RDF called a blank node. The blank nodes in figure 4 uses the relation sc:competencyDefinition for pointing out the item and the relation sc:competenceLevel to point out the item-value.

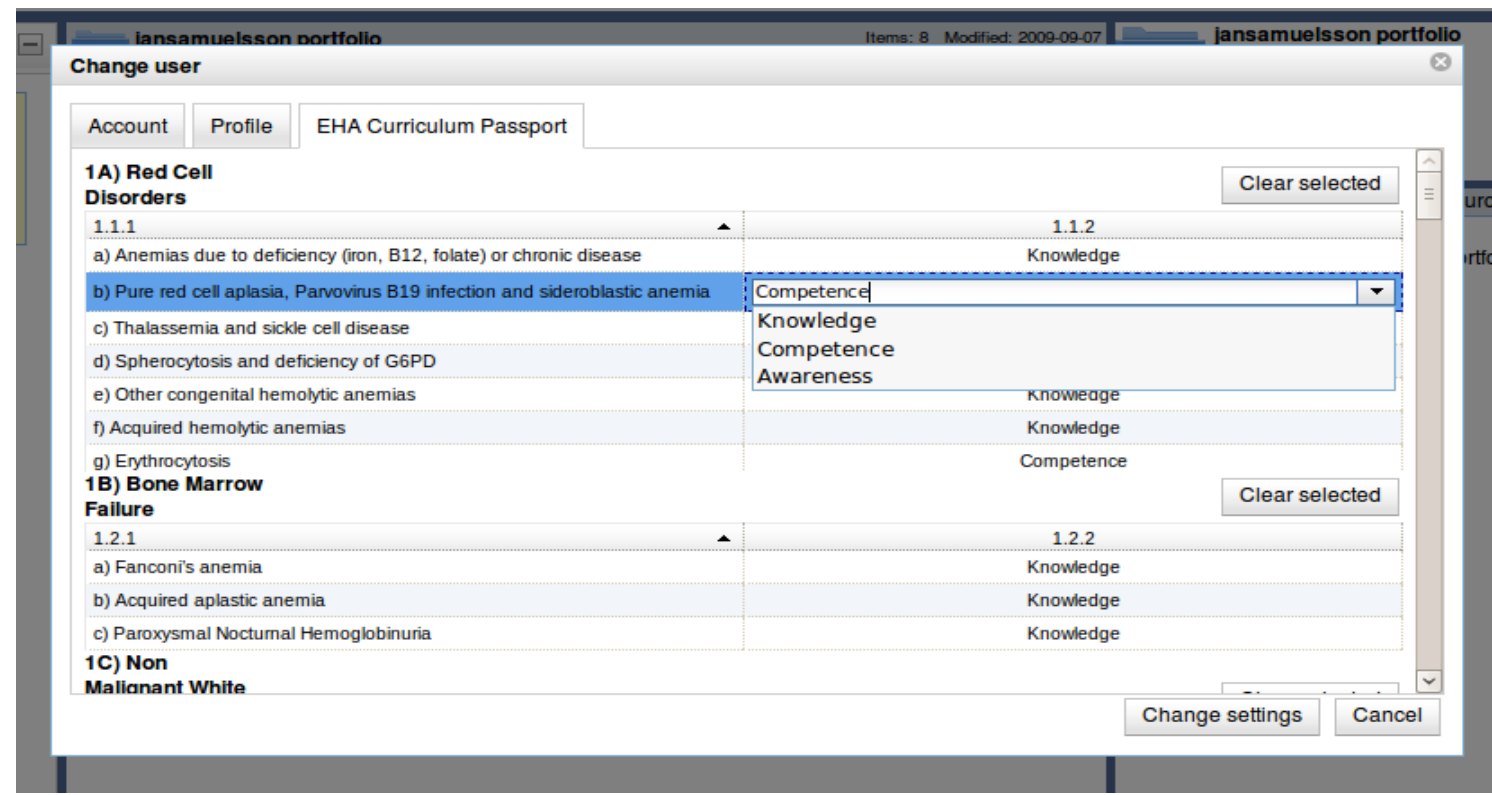

Figure 3. Screenshot of the CV-passport editor

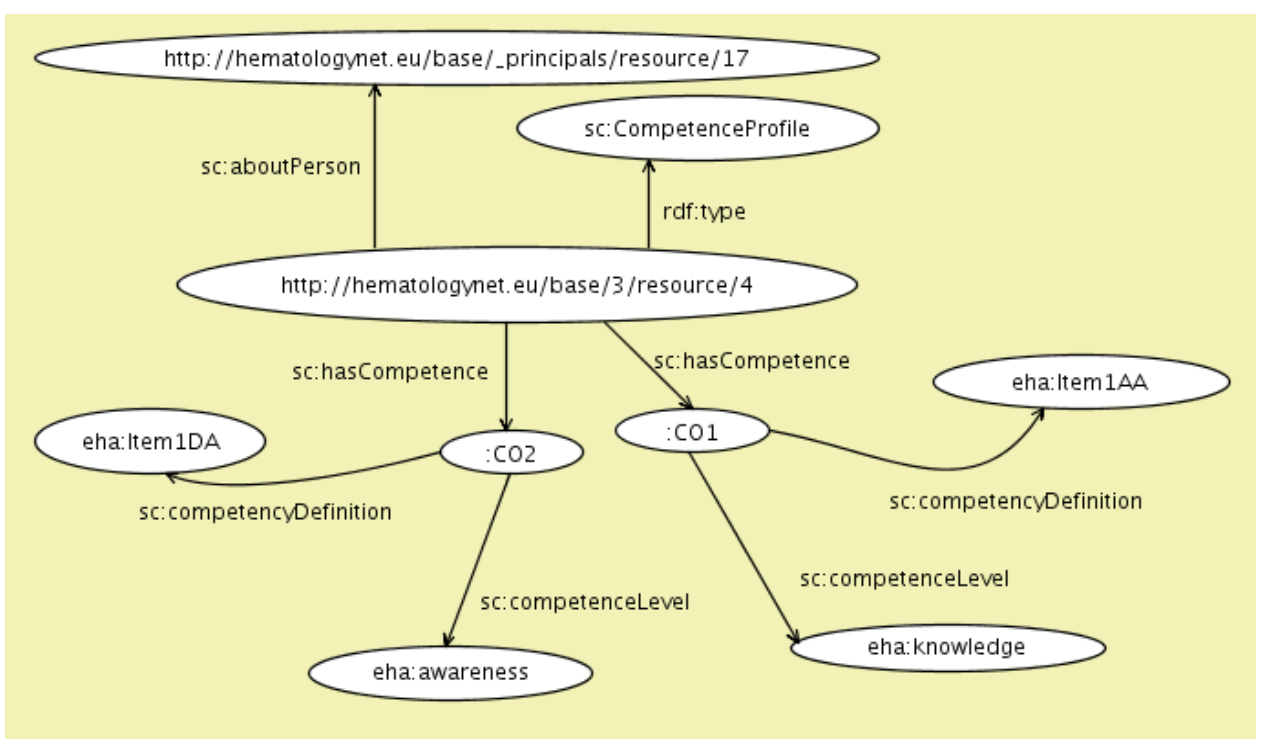

Figure 4. Competence profile in RDF for a user 


\subsection{Classifying Learning Resources}

One of the purposes of portfolio systems is to allow the user to upload and link to material and organize them in different ways. The system can be used for sharing material that is interesting for oneself and others, for example material that several users have created or contributed to. Most of the resources added to the portfolio-system created in H-net will be about hematology and can in most cases be classified according to how they relate to different parts of the CV-passport. This information can then be used to facilitate searches of learning resources. The information is expressed in the metadata of the resource in three possible ways with different granularities, by linking it to:

1. one or more subsections of the passport

2. one or more items in the CV-passport.

3. a combination of an item and:

- a recommended level for the user to make constructive use of the resource, or

- an expected level for the user after taking part of the resource

The options in points 1 to 3 are possible to use in combination. Points 1 and 2 are expressed by a direct relation to the subsection or item, which corresponds to point 6 in the list given at the end of chapter 6 , ie tagging of resources. Moreover, since each subsection and item is assigned a URI, this solution follows the principle behind Linked Data $^{1}$, which is the idea of linking data across the web. The use of point 3 is when any of the following can be expressed:

- $\quad$ The prerequisite competence/knowledge - This is the knowledge needed to actually make use of the resource in the way it was intended.

- The targeted competence/knowledge - This indicates that the resource can be considered useful for the learning process that is aimed at achieving the specified level.

Figure 5 shows both the metadata editor and how the data is structured. If the prerequisite competence/knowledge is edited, it should be interpreted as that the resources is aimed at an audience that for the passport item have reached that level. With a targeted competence/knowledge it is should interpreted that the resource is considered useful in the learning process of achieving a certain level for an item.

\subsection{Searching for learning resources}

Normal text-search is the most common way of searching on the web. This method can sometimes produce a lot of matching resources, and to resolve that problem each match gets a ranking that is done differently depending on the search engine. Some search engines keep information on how often a resource is clicked by the user when a certain word is in the search, others give a high ranking if the word appears many times and early

1 http://linkeddata.org/ 
in the text of the resource. Several other methods exist, but they usually have in common that they need to process the actual resource and/or store indexes.

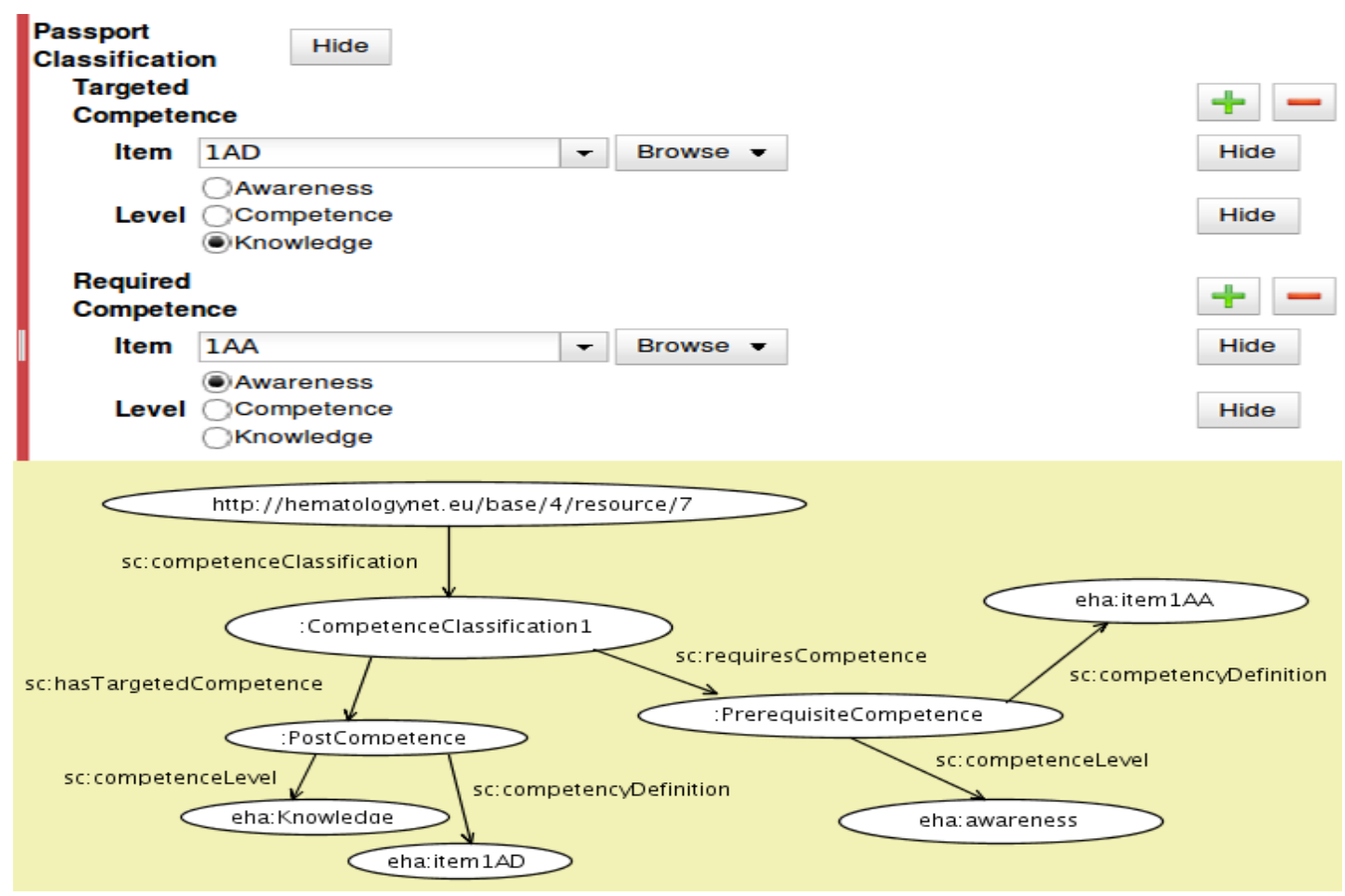

Figure 5. Learning resource with information about the required and targeted competence

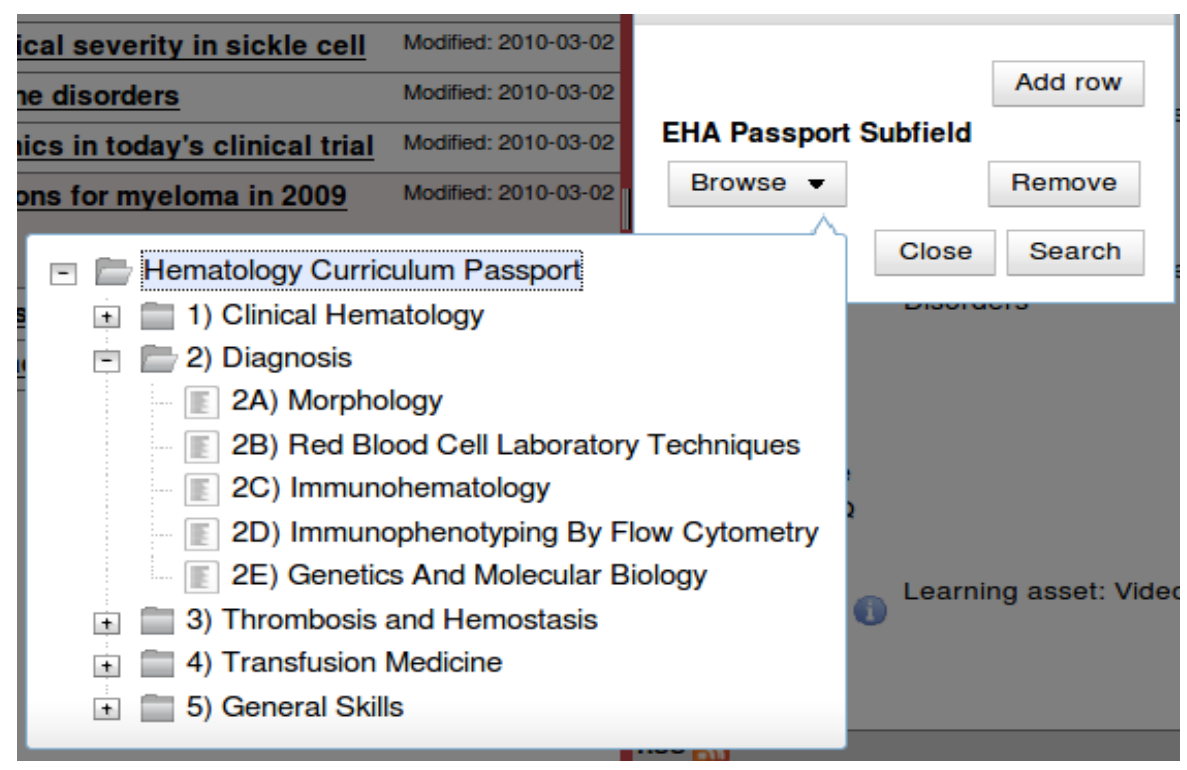

Figure 6. Search by subsection, the URI:s of the subsection are hidden from the user 
The approach in the portfolio system is to store structured metadata about the resource, instead of extracting it from the actual resource. In that way the resource and its description are kept separate. With the metadata on what sections or items in the passport a resource relates to, as described in chapter 2.3 , an alternative way to search by using the $\mathrm{CV}$-passport is enabled. A search can be performed with one or more sections or items as input, which are represented as a URI. This is not something that should be exposed to the user, instead the description for each should be used and the URI obscured. Figure 6 below shows how the user interface for this search in the portfolio system. By using the hierarchy of the CV-passport the user have the possibility to browse by expanding different sections.

With this type of input, the portfolio system can search those resources linked to the URIs it is provided with. If several URIs are provided the matches can include the resources where all URIs match, or the resources where any of the URIs match. Which option to choose could be given as a choice for the user in the user interface. If the number of URIs given to the search the number of matches decreases if they all need to match, but if only one of them need to match the number of matches will instead increase. Which one to use as the default option is not obvious.

As the system is also aware of what items a subsection holds, the search could be made even more complex by involving that information. For example, if the user searches for resources related to the subsection Red Cell Disorders, the search will also match resources that are tagged to be about item $1 \mathrm{AA}$ as that item is a part of the subsection (see figure 1).

\subsubsection{Personalised search}

Another possible search utilizing the CV-passport involves learning resources that are described with the purpose to be used in the learning process of achieving a specific competence, which in this context corresponds to achieving a certain level for one or more passport items. The concept behind this kind of search was given by Naeve et al (2008) where competence is considered as being a way to express a learning goal of a learning process and a Learning Resource can be connected to such a goal, by defining a targeted competence. In that way it is given a specific purpose, ie helping the user to achieve a certain competence.

In this kind of search the input is a selected set of items where the goal level is specified together with the user's CV-passport. The search is performed by:

1. Find all the learning resources that have the items in their targeted competence classification connected to one of the items in the search.

2. Check the levels that is targeted and sort out the ones that have the level specified according to the search. It could be a good idea to save the rest of the matches in step 1 in case there are few exact matches.

3. Check the required competence classification in the matches sorted out after step 2. In case they, for an item specified, have the same level as in the users CVpassport, then such resources are considered to be a better match and should be rated higher. 
If the search was for Learning Resources that were described with the purpose of achieving the level "competence" for item $1 \mathrm{AD}$, the example resource in figure 5 will match in step 1 above. It will not pass step 2, but it could still be useful for the user. With a few exact matches, the resources sorted out at step 2 can be presented to the user as not being exact, but it could be used for achieving a part of the goal. The same principle applies in step 3: The resources that have the same required level for an item as the user has in his/her CV-passport are considered a better match. But the ones that do not should be kept and presented to the user as a match that is not exact.

\section{Interoperability with other medical specialities}

As stated previously, the medical field of hematology is closely related with other medical fields. So, it is suitable if the data in the portfolio system and the data structures used can be reused for applications used by communities in those fields. This has been an important aspect when the design choices of the portfolio system and the underlying representation of data have been made. One important design choice for achieving this was that the subsections of the passport stores the information of what items it has as members. That means that the items are not tied with their descriptions in a specific passport and any other passport can have subsections that point out any item. A problem that occurs when reusing items in that way is if the owner of a item (in this case the EHA) decides that the item is deprecated and will be removed. How is this communicated to the the application using the item? How to deal with this is a problem that is possible to solve inside the portfolio system, but depending on how this is done, it can have implications on other applications reusing the data. When using RDF, there is not yet a recommended solution for this that can be applied, so until then the owners of external applications needs to be notified in a semi-automated way.

\subsection{Flexible annotation of metadata}

With a solution for deprecating items, the editors that are using the data in the passport needs to be changed as well. Fortunately, the portfolio-system creates those editors from a configuration that uses the concept of Annotation Profiles (Palmér, Enoksson, Nilsson and Naeve) where it is described what metadata can be edited and also in what way. This mechanism can be used for creating editors for RDF metadata and when the CV-passport is updated, only the configuration needs to be updated. Thus, there is no need to change the actual code of the portfolio system.

The CV-passport has its section and subsections which are recreated in the Annotation Profile, as can be seen in the editor in figure 3. However, the underlying data stored only connect the user to the items and the level. Since the editor can be changed by a new configuration, this can be one way of reusing the items of the passport into passports for other specialities. Ie, an editor for a second passport uses some items from the hematology CV passport. Moreover, if certain items are not considered necessary within an organisation, country or region, they can choose to have their own editor with an Annotation Profile where those items are excluded.

\subsubsection{Customized CV-passport}

As the usage of items are designed to be rather flexible, it would even be possible for the users to make their own CV-passport just by combining a customized set of items from 
the different $\mathrm{CV}$-passports that exists. In that way the user can set up his/her own learning goals by using a self-selected combination of items.

It can be discussed whether this is desired and useful, but it would be a challenge to manage this information and create good graphical user interfaces that dealt with this in a way that would be easy to understand. That could also be a typical example of an external application that is using the data or the data structure in the portfolio system.

\subsection{The need for a common competence model}

As of now a rather simple model for competences has been used. Each item of the passport is classified as a competency definition (see figure 2). Furthermore, it is possible to connect the item to an item-value that tells the level for an item. How the items, itemvalues and competency descriptions fit into a larger context will be needed to be modelled further in order to see if they are compatible with the competence models of other possible CV-passports. For example, another passport might divide the competence into sub-competences, or use a different set of item-values.

The core part of how the competence have been modelled by Schmidt and Kunzmann (2006) fits rather well into the model in the CV-passport. A competency can use a set of CompetencyLevels, in a similar fashion as the items can be connected to the item-values. Other approaches do exist like HR-XML ${ }^{1}$ and $\mathrm{RCDEO}^{2}$ and lately the EUfunded project eCOTOOL (http://www.ecompetence.eu/) which has as a long-term goal to develop a European skills and competence model. The competence model for the CVpassport will need to be in to some kind of general model, and that is one the future work that lies ahead with the web-version of the passport.

\section{Conclusion and challenges ahead}

A curriculum of hematology (the CV-passport) developed for and by the hematology community has been given an RDF representation and can thus work in a web environment. The possible uses of that representation are many and some important examples have been given here. The original intention was to have it as a document that describes the knowledge/competence of a hematologist by setting the appropriate level for each item. When filling out the digital CV-passport, the hematologist will do self evaluation of their competence, which has proven to not always reflect the actual competence/knowledge (Davis, Mazmanian, Fordis, Van Morrison, Thorpe \& Perrier , 2006). To have some kind of way for a mentor/senior hematologist to confirm the filled out CV-passport would definetly be useful in future versions.

The parts of the CV-passport have also been used for describing learning resources by linking them to one or more subsections or items. That way the tags added to the resource comes from a curriculum that is well-known by the user and that information is then also used for searching after learning resources. Furthemore, the actual purpose of a learning resource, ie achieving a certain level for an item, can also be described in the metadata. This kind of information is then used for an alternative and personalised way of searching, that also includes the users current competence by having the filled out passport included in the search.

1 http://www.hr-xml.org

2 http://www.imsproject.org/competencies/ 
The results of H-net presented here also opens up for other specialities to reuse the way to structure the data and also to reuse the actual items of the CV-passport. That way the passport does not need to be living in isolation, but can instead be used its item can be used in different combinations in a rather free manner. This comes also from conscious choice of technology that is and will be used in the future of the web. The use of editors that are easy to change, by the use of Annotation Profiles, also enables the possibility to create specific passport editors for a region, country or even a editor created by the user. However, the competence model of the CV-passport needs to be modelled in more detail, so that it can be easily judged if two passports uses a similar model and can be used in conjunction.

It should also be mentioned that the American Society of Hematolgy (http://www.hematology.org/) also have a curriculum for hematology (Gitlin \& Melnick, n.d.), but it is aimed at just being a curriculum. It is described what a hematologist ought to be skilled in, but the user of it cannot give a current level and it cannot in an easy way set up goals for personal development as in the EHA passport.

\section{References}

1. Bulgarian Society of Clinical and Transfusion Haematology. (2005). European Haematology Association Curriculum Project - Version 2005. Retrieved July 8, 2010 from http://www.bscth.med-bg.info/site/eng/prodmedobuch.

2. Davis, D.A., Mazmanian P.E., Fordis M., Van Morrison, R., Thorpe, K.E. \& Perrier, L. (2006). Accuracy of physician self-assessment compared with observed measures of competence. A systematic review. Journal of the American Medical Association, 296(9), 1094-102.

3. Ebner, H. \& Palmér, M. (2008). A mashup-friendly resource and metadata management framework, In Wild, F., Kalz, M., and Palmér, M. (eds.) Proceedings of the First International Workshop on Mashup Personal Learning Environments (MUPPLE08), Maastricht, The Netherlands, September 2008, (pp. 48-56). CEUR Workshop Proceedings vol. 388, 2008.

4. EHA (European Hematology Association). (2006). Mission Statement European Hematology Association. EHA Executive Office. Retrieved July 11, 2010 from http://ehaweb.org/content/download/1205/7083/file/MISSION\%20STATEMENT \%20EUROPEAN\%20HEMATOLOGY\%20ASSOCIATION\%202007.pdf.

5. European Hematology Association. (2005). European Hematology Curriculum/Passport. EHA Executive Office. Retrieved July 13, 2010 from http://www.ehaweb.org/media/files/education/european_hematology curriculum_p assport.

6. European Hematology Association. (2008). European Council for Accreditation in Hematology. Leonardo Da Vinci National Agency/ European Comission. Retrieved July 9, $2010 \quad$ from http://www.marathonmultimedia.com/cme/71452\%20EHA\%20Brochure $\% 20 \mathrm{bw} \%$ 20(gr).pdf.

7. Fielding, R.T. (2000). Architectural Styles and the Design of Networked-based Software Architectures, Chapter 5: Representational State Transfer (REST) (Doctoral Dissertation). University of California, Irvine. Retrieved July 15, 2010 from http://www.ics.uci.edu/ fielding/pubs/dissertation/rest_arch_style.htm. 
8. Gitlin, S.D., \& Melnick, A. (n.d.). American Society of Hematology, Hematology Curriculum. American Society of Hematology (ASH). Retrieved July 15, 2010 from http://www.hematology.org/Training/Directors/4756.aspx.

9. Hematology. (2003). MedTerms MedicineNet WebMD network. Retrieved July 10, 2010 from http://www.medicinenet.com/script/main/art.asp?articlekey=12510.

10. Hematology. (2006). WordNet 3.0 Princeton University. Retrieved July 9, 2010, from http://wordnetweb.princeton.edu/perl/webwn?s=hematology.

11. Miller, E., \& Manola, F. (2004). RDF Primer. World Wide Web Consortium (W3C). Retrieved July 14, 2010 from http://www.w3.org/TR/rdf-primer/.

12. Naeve, A., Sicilia, M.A., \& Lytras, M.D. (2008). Learning processes and processing learning: from organizational needs to learning designs. Journal of Knowledge Management, 12(6), 5-14.

13. Palmér, M., Enoksson, F., Nilsson, M., \& Naeve, A. (2007). Annotation profiles: configuring forms to edit RDF. In Proceedings of the 2007 international conference on Dublin Core and Metadata Applications, (pp. 10-21). Singapore, Dublin Core Metadata Initiative.

14. Schmidt, A. \& Kunzmann, C. (2006). Towards a Human Resource Development Ontology for Combining Competence Management and Technology-Enhanced Workplace Learning. In Meersman, R., Tahiri, Z. and Herero, P. (ed.) On The Move to Meaningful Internet Systems 2006: OTM 2006 Workshops. Part I. (pp. 1078-1087), Springer.

15. Summers, E. \& Isaac, A. (2009). SKOS Simple Knowledge Organisation System Primer. World Wide Web Consortium (W3C) Retrieved July 15, 2010 from http://www.w3.org/TR/skos-primer/.

16. Van Assche, F. (2007). Linking learning resources to curricula by using competencies. In Massart, D., Colin J.N. Van Assche, F. (Eds), Proceedings of the First International Workshop on Learning Object Discovery and Exchange, LODE 2007, Sissi, Lassithi -Crete, Greece (pp. 80-90) CEUR-WS.org. 